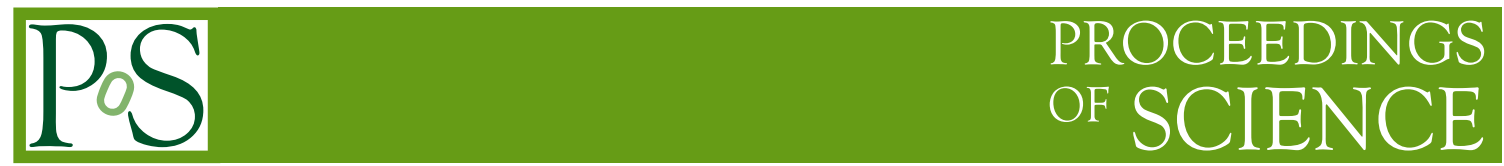

\title{
Lattice Simulations using OpenACC compilers
}

\author{
Pushan Majumdar* \\ Indian Association for the Cultivation of Science, Kolkata \\ E-mail: tppmeiacs.res.in
}

OpenACC compilers allow one to use Graphics Processing Units without having to write explicit CUDA codes. Programs can be modified incrementally using OpenMP like directives which causes the compiler to generate CUDA kernels to be run on the GPUs. In this article we look at the performance gain in lattice simulations with dynamical fermions using OpenACC compilers.

31st International Symposium on Lattice Field Theory - LATTICE 2013

July 29 - August 3, 2013

Mainz, Germany

*Speaker. 


\section{Introduction}

Graphics Processing Units (GPUs) are increasingly turning out to be the hardware of choice to run the compute intensive parts of lattice simulations since they have about an order of magnitude better price-performance ratio compared to normal CPUs. Additionally they consume only a fraction of the power for the same computing capacity.

Until recently, programs capable of running on GPUs had to be written in special languages (for example CUDA[1], which is like an extended version of $\mathrm{C}$ appropriate for the GPU) or even assembly level languages. A library of CUDA routines necessary for simulating lattice QCD is available [2]. However the need of a different language created a large barrier for a lot of people trying to use the GPUs.

During the last two years there has been a development of directive based compilers called OpenACC compilers for both FORTRAN and C (much like OpenMP compilers) which allow programs written in standard FORTRAN and $\mathrm{C}$ to use the GPUs. In this article we report on our attempt to run lattice QCD simulation on GPUs using the Cray OpenACC FORTRAN compiler.

In section 2 we give a very brief introduction to OpenACC. Section 3 describes the matrixvector multiplication routine which is called many times inside the conjugate gradient routine. Section 4 outlines the conjugate gradient subroutine on a single GPU while the next section points out the differences necessary for the multi-GPU version. Finally in section 6 we draw our conclusions.

\section{OpenACC}

OpenACC is a programming standard for parallel computing developed by Cray, CAPS, Nvidia and PGI. The goal of the standard is to simplify parallel programming of heterogeneous CPUaccelerator systems [3]. The OpenACC Application Program Interface (API) lists a collection of compiler directives to specify loops and regions of code in standard C, $\mathrm{C}++$ and Fortran programs which should run on an attached accelerator. The API is designed to provide portability across operating systems, host CPUs and accelerators. The directives and programming model defined in this document allow programmers to create high-level host+accelerator programs without the need to explicitly initialize the accelerator, manage data or program transfers between the host and accelerator, or initiate accelerator startup and shutdown [4].

The different simulation programs we have had some experience with are :

(i) staggered fermions with the Wilson gauge action on both single and multiple GPUs,

(ii) Wilson fermions with the Wilson gauge action on a single GPU.

In the OpenACC programming model, even though the actual movement of the data is done automatically by the compiler, the programmer has to control the movement of the data carefully between the CPU and the GPU. This is crucial for efficient running of the program. The reason is the slow data movement between the CPU and the GPU. While the speed with which data is moved from the main memory of the computer to the CPU (with say 16 cores) is anything between 25 and $30 \mathrm{~GB} / \mathrm{s}$, the speed between the main memory and the GPU (with > 500 cores) which sits in a PCI slot is only about $5 \mathrm{~GB} / \mathrm{s}$. The result is if one is not careful with the movement of data, the GPU cores would spend most of their time waiting for the data. 
One option would be to bypass the CPU completely and do the computation entirely on the GPU. However as of date it is impossible to do so as the GPU is incapable of handling input-output. Also in the openACC programming model, 'if-then' clauses are evaluated on the CPU. Moreover if one uses BLAS functions, they are launched from the CPU and MPI calls (at least for Fermi GPUs) are also launched from the CPU.

\section{Matrix-vector multiplication routine}

In this section we point out the differences between the openMP and openACC versions of the matrix-vector multiplication routine. One error we encountered with the presence of data while porting this routine to the GPU might be worth pointing out. The data is copied from the main memory to the GPU at the start of the conjugate gradient program. The matrix-vector multiplication routine uses the same data. Nevertheless we observed that if the compiler does not inline the routine, the matrix-vector multiplication routine does not find the necessary data. One simple way to avoid this problem is of course to inline the routine by hand, however that makes the program less modular and legible.

Below we list the differences between the openMP and openACC versions of the routine.

$$
\begin{gathered}
\text { subroutine matrix-vector (offset, local_length, } \mathrm{v}, \mathrm{w} \text { ) } \\
\text {...All kinds of definitions and declarations... }
\end{gathered}
$$

Original variable declaration in openMP program

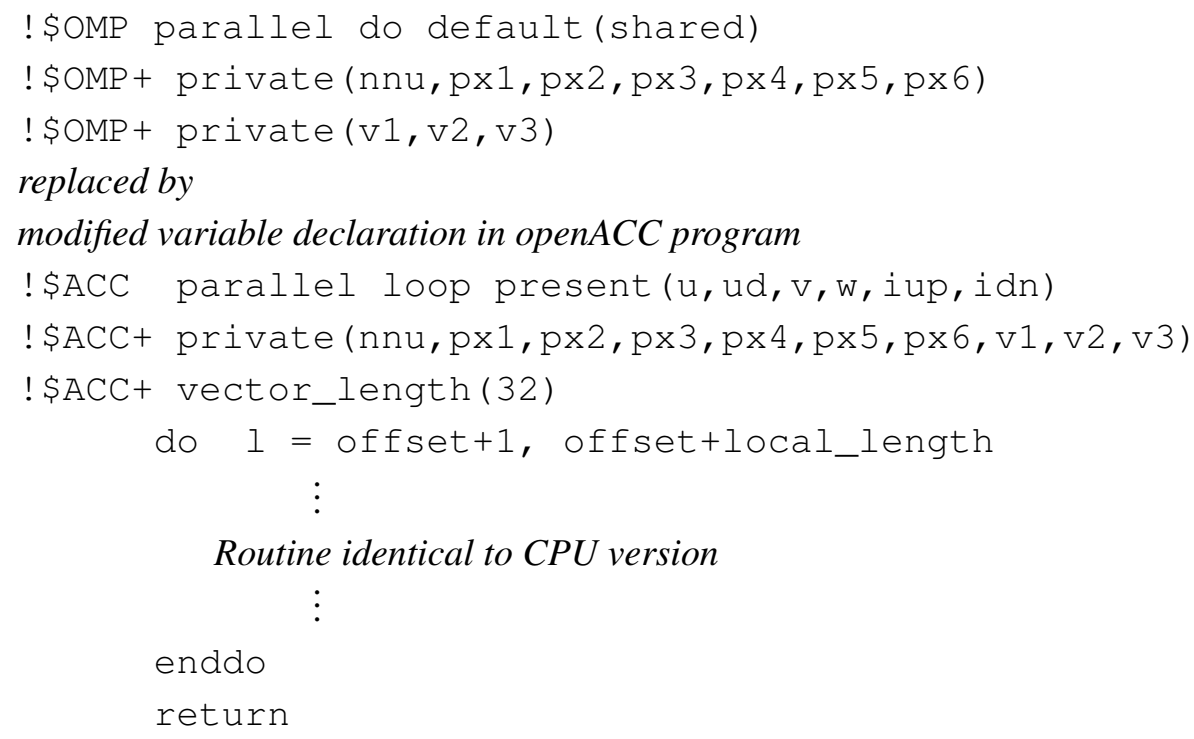

The compiler generates the CUDA kernel for the loop immediately following the directives and launches it on the accelerator. The qualifier present ( $u, u d, v, w, i u p, i d n)$ tells the compiler that the arrays $u, u d, v, w, i u p, i d n$ are already present on the accelerator. Another possible option is to use present_or_copy.

\section{Single GPU conjugate gradient routine}

In this section we outline the openACC version of the conjugate gradient routine which is the 
main work horse of the simulation program and takes between $80-90 \%$ of the computing time. Therefore porting this routine to the GPU is essential for maximizing the gain from the GPU.

The GPU portion of the code starts with a data directive and ends with a end data directive. Between these one can use other directives like copy, copyin, copyout, create to move data between the CPU and the GPU or define variables on the GPU. As implied by the name, copy in only copies the data into the GPU, copyout copies the data from the GPU memory to the CPU memory, copy copies the data from the CPU memory to the GPU memory at the beginning of the data section and copies the updated value back to the CPU memory after the end of the data section. create defines the variables only on the GPU.

In the following code segment (in fortran) we often use $\langle x, y\rangle$ to denote the inner product between $\mathrm{x}$ and $\mathrm{y}$ instead of writing out the loops.

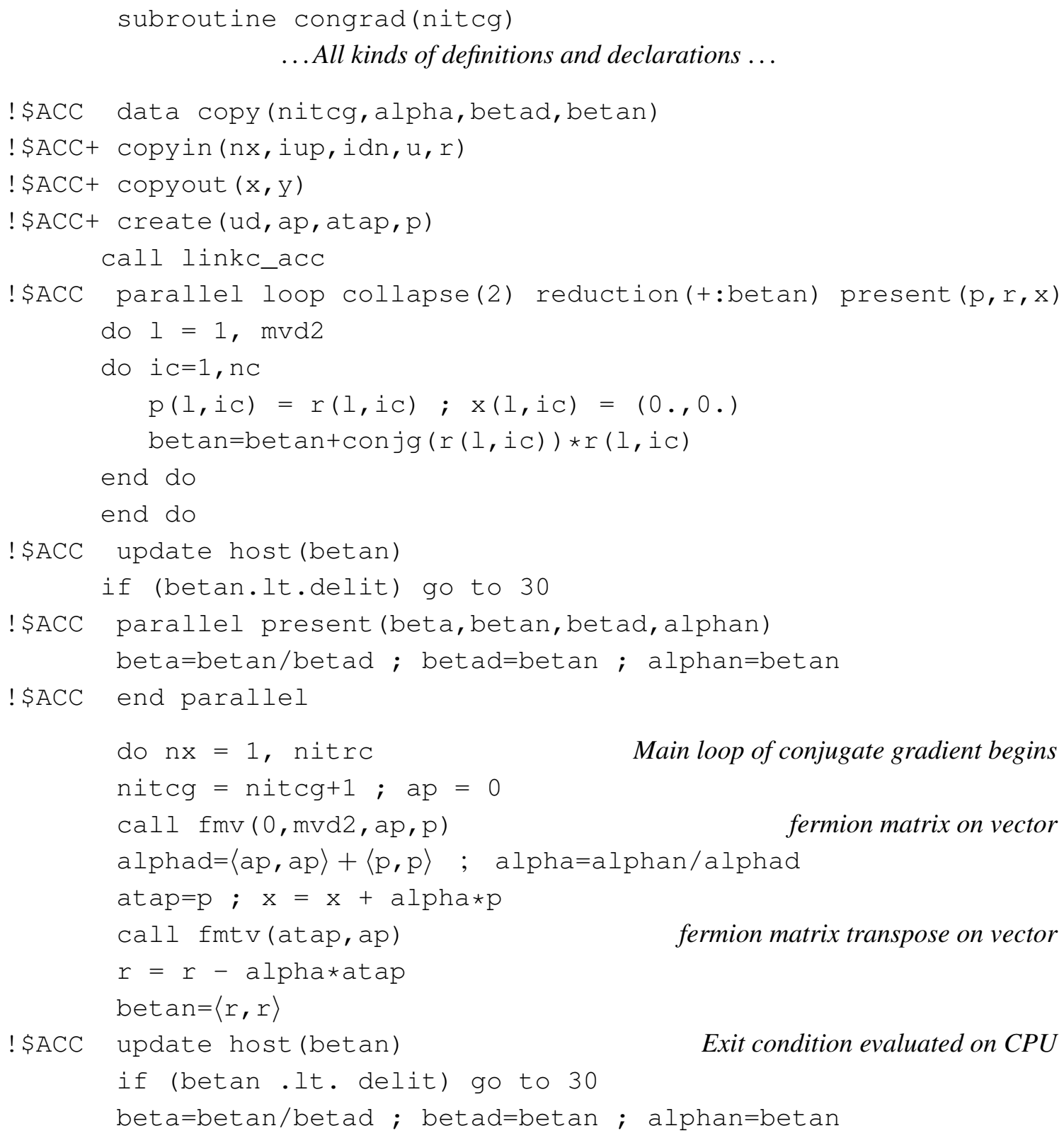




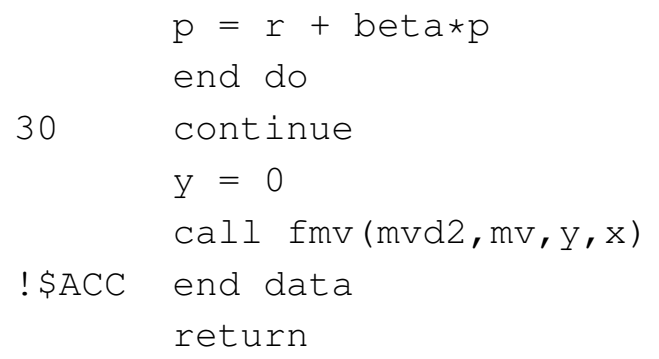

Main loop of conjugate gradient ends

Solution on the second half lattice fermion matrix on vector

The first new construct we encounter is the collapse $(n)$ directive. This directive directs the compiler to collapse the $\mathrm{n}$ nested loops into a single parallel loop and create the kernel for that. For reduction operations, we need to specify the reduction variables to avoid error building.

The next new directive is upadte host. As we have mentioned previously, the 'if-then' branching conditions are evaluated on the CPU in the openACC programming model. Therefore if a variable is updated in the GPU memory and will be used in an 'if-then' condition, then the updated variable has to be copied to the main memory. The update host directive does just that. It updates the value of the variable in the argument of update host in the main memory.

Even inside a data region, kernels for the GPU are created only when statements are within the parallel and end parallel directives. Otherwise they are evaluated on the CPU. We find that the program runs faster if we give up the fortran vector and array constructs and perform operations on arrays using parallel loops. Our observation is that calling BLAS functions slows down the program a lot as they are launched only from the main memory. We therfore do all the copy and reduction operations using loops.

The compiler options (specific to the Cray openACC compiler) we used for the GPU portion are -hacc_model=fast_addr:auto_async_all.

\section{Multi-GPU conjugate gradient}

In this section we point out the changes that have to be made in the conjugate gradient and the matrix-vector multiplication routines (which in this case is manually inlined in the conjugate gradient) for the program to run on multiple GPUs which are on different nodes of a cluster and therefore have to use MPI calls to communicate between them.

For simplicity, we assume in the following that only the fermion matrix on vector multiplication is split between the nodes so that the output vector denoted by ap in the previous section is replaced by ap_loc and that needs to be communicated to the other nodes for constructing the full vector which will then serve as the input vector for the fermion matrix transpose on vector multiplication.

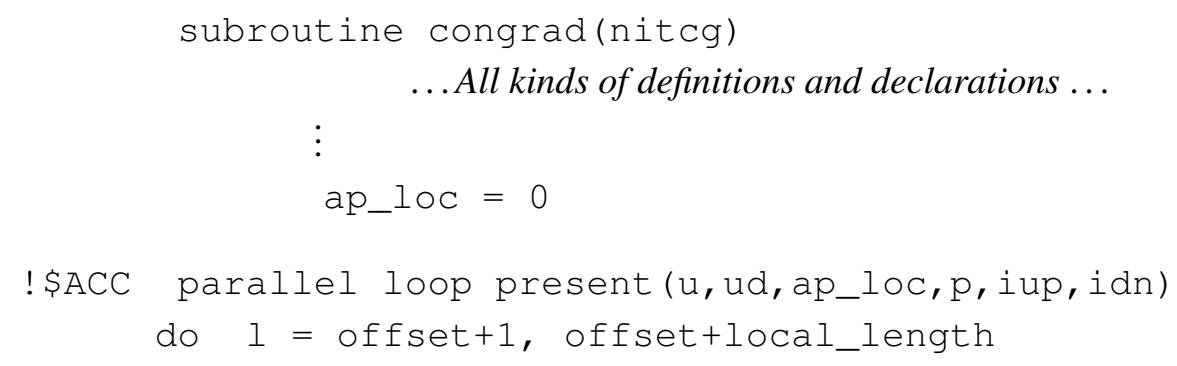




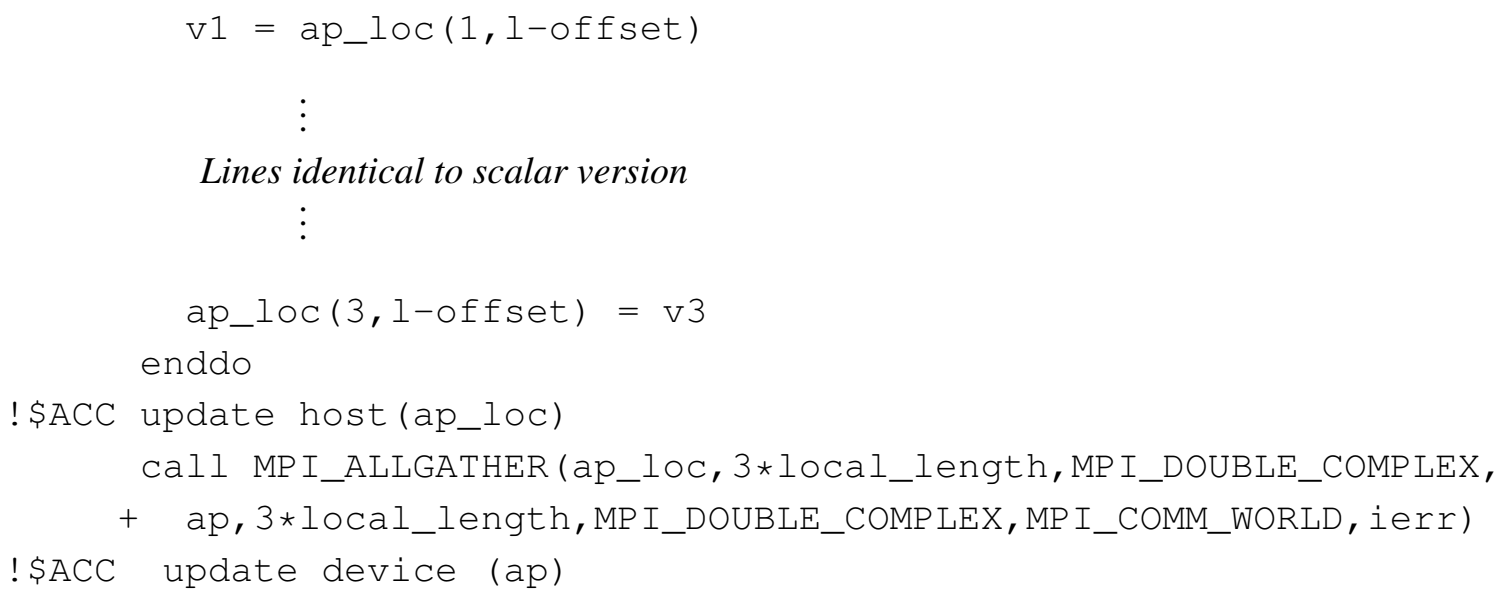

The new directive here is the update device. After the local multiplication is done, the output is moved to the main memory by the update host (ap_loc) directive. Then the MPI_ALLGATHER is launched from the CPU to create the full input vector ap. This updated ap now has to be moved from the main memory to the GPU memory. This is achieved by the directive update device (ap).

The compiler option for the multi-GPU routine is slightly different. Instead of auto_async_all we need to use auto_async_kernel as otherwise the order of operations of update host, MPI_ALLGATHER and update device will not be maintained.

\section{Performance \& Discussion}

In figure 1 we compare the performance of a $N_{f}=2$ staggered fermion simulation program (Hybrid Molecular Dynamics) on a cluster, a Cray XE6 and X2090 GPU on one node of a Cray XK6. The performance of single GPU staggered fermion code is roughly equivalent to 128 cores of cluster with QDR infiniband interconnect and slightly more than 32 cores of Cray XE6.

We also did some preliminary runs with a $N_{f}=2$ Wilson fermion program (Hybrid Monte Carlo). That gave a performance equivalent to about 96 cores of the cluster. For the Wilson fermion case, we have a hand coded CUDA program which is about $30 \%$ faster than the openACC program.

Even without additional storage optimizations like storing only two columns of the gauge fields, a GPU with 6GB memory fits in a $32^{4}$ Wilson fermion lattice or a $10 \times 40^{3}$ staggered fermion lattice.

To summarize, in openACC programming, the coding effort is only marginally higher than OpenMP. Almost each OpenMP directive can be replaced with a OpenACC directive. The only additional structure is the creation of a data region with a list of variables (scalars and arrays) so that the compiler knows which variables to copy to the GPU and back again. Since the connection between the GPU and main memory is comparatively slow, the data flow between the CPU and the GPU needs to be minimized. In fact we find an appreciable gain in performance only when the entire conjugate gradient routine runs on the GPU.

Fermi GPUs cannot issue MPI calls. So for Multi-GPU programs using MPI, every MPI call involves a data transfer from the GPU to CPU and back. Each such copy adds a significant $(\sim$ 


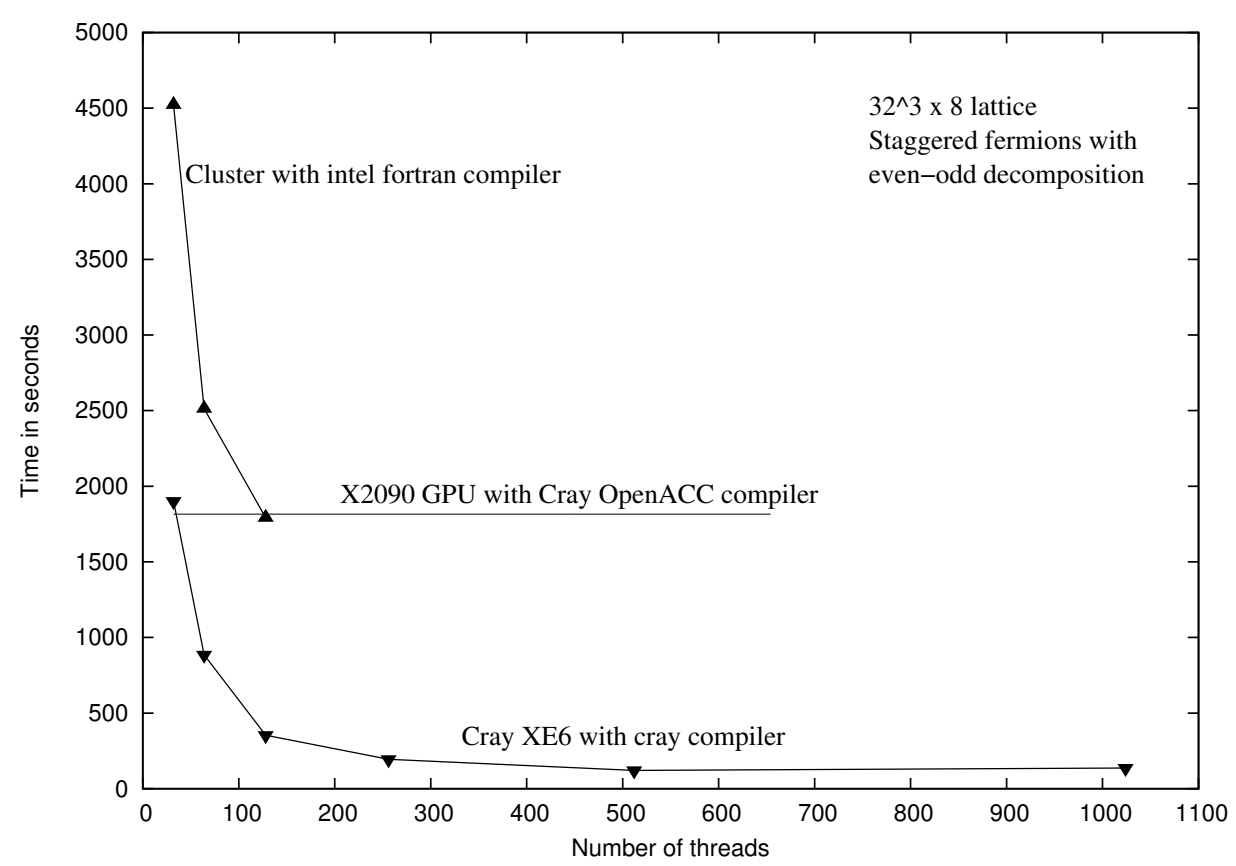

Figure 1: The figure compares the performance of a staggered fermion simulation program on a cluster $(2.2$ $\mathrm{GHz}$ Opteron (6 core) with QDR Infiniband interconnect) with the intel fortran compiler, a Cray XE6 (2.1 $\mathrm{GHz}$ Opteron (16 core) with Cray interconnect) with the cray fortran compiler and a single X2090 (Nvidia Fermi GPU) using the Cray OpenACC compiler.

15\%) overhead to the runtime. For Kepler GPUs the construct host_data use_device cuts this down to a certain extent. Nevertheless the timings we obtain show that GPUs are extremely powerful tools if one does not have access to conventional supercomputers.

\section{Acknowledgments}

The author would like to acknowledge ILGTI-TIFR for funding the GPU portion of the Cray on which these studies were carried out and IACS for funding the rest of the machine. The author would also like to acknowledge the DST grant SR/S2/HEP-35/2008 for funding the cluster for the comparison runs and on which the hand coded CUDA program was developed. Finally the author also acknowledges the Cray India team for help at various stages during the development of the OpenACC codes.

\section{References}

[1] http://www.nvidia.com/object/cuda_home_new.html

[2] M.A. Clark, R. Babich, K. Barros and C. Rebbi, Solving Lattice QCD systems of equations using mixed precision solvers on GPUs, Comput. Phys. Commun., 181 (2010) 1517,

[arXiv:0911.3191]; http://lattice.github.io/quda/

[3] http://en.wikipedia.org/wiki/OpenACC

[4] http://www.openacc-standard.org/ 